\title{
The Pathophysiology and Care of Exercise Related Muscle Cramps
}

\author{
${ }^{1 *}$ Abhay Kumar Pandey, ${ }^{2}$ Abha Pandit, ${ }^{3}$ Bajarangprasad L Pandey
}

\author{
${ }^{1}$ Department of Physiology, All India Institute of Medical Sciences, Bhopal, India \\ ${ }^{2}$ Department of Medicine, Index Medical College Hospital and Research Centre, Indore, India \\ ${ }^{3}$ Department of Pharmacology, Institute of Medical Sciences, Banaras Hindu University, \\ Varanasi Program in Biomedical Science, India
}

\begin{abstract}
Cramps are major concerns to competing athletes occurring during or after exercise, are common yet, poorly understood phenomena. Pain alone is not object of treatment as serious musculophysiologic and metabolic disturbance of fluid and electrolyte deserve correction. Acute muscle pain and stiffness may cause soreness for longer time. Based on observations, two etiological theories are construed, i.e. the muscle fatigue theory and sodium-water deficit theory. Either has supporting and contradicting facts, but these are relevant to guide prevention and management interventions. Cramps may be different in kind based on different local and/or general causes. Occurrence of cramps in varied situations, environmental conditions and populations, suggests of pleural causal determinents. These include neuromuscular and fluid-electrolyte disturbance factors directly responsible under specific circumstances of individual sports person. Degree of conditioning to particular kind of physical exertion appears most significant factor. Prevention exercises target theorised physiology of muscle tendon and golgi organ receptors, toward delaying fatigability and cramp risk. Occurrence of cramps mostly in hot environments emphasizes support to dehydration-electrolyte imbalance theory. Maintenance of hydration and adequate electrolyte levels in cramp-prone individuals thus makes sense. Worth of variety of measures empirically employed for cramp relief can be judged by scientific understanding. Drugs found useful may not be the best match to pathophysiologic proprieties and thus irrational. The pathophysiological details and relevant clinical information is presented and discussed as first hand understanding for the sports persons and their care givers.
\end{abstract}

Keywords: exercise cramps, heat exhaustion, muscle fatigue, sports medicine, sweat loss

Athletes commonly suffer muscle cramps that create fear of appearing without warning or apparent cause during competition. Exercise related muscle cramps are painful spasmodic involuntary contraction of skeletal muscle that occur during or immediately following extensive exercise. The musculoskeletal dysfunction may persist to compromise competitive performance besides leading to muscle damage [1]. Cramps may often localize in over worked, fatigued muscle due to inadequate conditioning. More generalised exertional heat cramps occur due to excessive sweating and sodium loss during or after exercise. Distinction between the two is crucial toward appropriate preventive and alleviating strategies. Prominent two of the proposed causal theories of

${ }^{*}$ Corresponding author:

Abhay Kumar Pandey

Department of Physiology,

All India Institute of Medical Sciences, Bhopal, MP (India)

Phone: +91-7607980255, Email: abhay.aslp@gmail.com cramping are muscle fatigue or disrupted neuro muscular control and the sodium deficit-dehydration theory that have guided prevention and treatment efforts [2].

\section{FATIGUE CRAMPS}

As a distinction, fatigue cramps are localized to overloaded and fatigued muscle group; and sometimes spread slowly across the involved muscle region without jumping [3]. The local fatigue implies an excitatory alteration (increase) in afferent activity in muscle spindle. There is concomitant decrease in inhibition in Golgi tendon organ, resulting in uncontrolled sustained activity of $\alpha$-motor neurons [2, 4-6]. Inhibitory neural mechanism of muscle contraction, when tendon organ senses increased muscle tension, is disrupted. Enhanced excitatory activity in muscle spindle triggers unopposed sustained involuntary muscle contraction, without control of tendon organ. Altered depolarization threshold at neuro-motor endplate results in vul- 
nerability to cramp [7], as inhibitory activity of golgi tendon organ is low in shortened position of muscle [4]. Primary factors in development of fatigue are intensity and duration of exercise, muscle contraction under shortened state and possibly, tissue damage [8]. Old age, poor stretching habit, insufficient conditioning, history of cramping, excessive exercise duration and intensity and related metabolic disturbances, are all predisposing risk factors $[6,9)$. Study in humans showed higher baseline EMG activity in cramping muscle in comparison to non cramping muscle [10]. Central as well as peripheral origins of the altered neuromuscular control have been proposed [1113].

\section{CRAMPS DUE TO SODIUM DEFICIT}

Sizeable deficit of exchangeable sodium develops when sodium and chloride loss in sweat, exceeds salt intake [14]. About 20-30\% loss in exchangeable sodium associates severe muscle cramping [15]. The sweating rate, sweat sodium concentration (20-80 $\mathrm{mmol} / \mathrm{L}$ ) and dietary intake of salt, determine the rapidity with which cramps may develop. The sweat sodium concentration does not decease with decrease in whole body sodium and water content. Sweating rate also remains constant over long term physical activity. Even the serum sodium concentration is not allowed to fall due to changes in sweat gland behaviour or sympathetic nervous activity, which increases sweat sodium concentration [16]. Other electrolytes like calcium, potassium and magnesium are also lost in sweating. The cramp prone athletes however, characteristically show sodium deficit due to inadequate dietary intake [14].

Water from interstitial compartment shifts to intravascular compartment to compensate loss of plasma volume during exercise due to sweating. Interstitial fluid therefore, contracts with continued exercise [17]. This continues even after exercise, due to continued sweating and cooling of body [18]. Plasma osmolality and electrolyte concentration are maintained or slightly elevated. As exercise related cramps occur during endurance or prolonged exercise, they are suspected to result from deficit of hydration and electrolyte, especially, sodium $[19,20]$. Body does not store enough water for exercise. Fluid and electrolyte depletion sensitizes select nerve terminals [21]. Dehydration induced contracture of interstitial space, presses on the motor nerves that may culminate in cramp.

Braulick et al. [1], induced hypo-hydration with moderate electrolyte loss in subjects, but did not detect increased susceptibility to exercise related cramps. Cramp threshold frequency remained unchanged despite hypo-hydration. Ingestion of carbohydrate-electrolyte fluid matching the losses in sweat did not appear to protect in $69 \%$ of cramp sufferers following exercise, in another study [22]. Stretching of the affected muscle offers immediate relief, while fluid electrolyte status remains same [2]. Maintenance of fluid and salt balance during exercise in hot environment however, is clearly relevant to prevention of heat cramps [14].

The type and the amount of fluid taken by the athlete however, may change electrolyte and fluid shift [23]. At a level of dehydration, lowered plasma sodium concentration and continued high sodium sweating would lower osmotic drive of plasma and delay maintenance of plasma volume through fluid shift [24]. In such state, low sweating rate will cause significant sodium deficit and risk of cramp only on extended period of exercise. High rate of sweating would accelerate loss of plasma volume and cause muscle cramping [25]. These athletes are 'salty' sweaters and are at high risk of rapid development of exertional heat cramps [26]. Other athletes may suffer similarly, if their sweating rate is high and exercise duration is prolonged [27].

Contraction of interstitial fluid compartment would cause mechanical distortion of some neuromuscular junction and the unmyelinated nerve terminals. The synaptic membrane would face more concentrated exercise-related metabolites, electrolytes and acetylcholine in extracellular milieu. These may cause the nerve fibers to independently mount end plate excitatory post synaptic potential, or fire [21,28]. Surface electromyographic analysis has verified occurrence of action potentials initiated from axon terminals of alpha-motor neurons during cramping [3]. Further contraction of interstitial fluid compartment would spread such exaggerated excitability among surrounding muscles resulting in jump of the cramps [29].

\section{APPLIED PERSPECTIVES}

Overload and fatigue related local cramps may resolve with passive stretching, massage, active antagonist muscle contraction or ice pack on affected muscles. Proper exercise training and conditioning as well as reduced exercise load and duration help to avoid these [30,31]. Fatigue cramps would not permit continued activity. Stretching at full length with afflicted muscle group, can restore the physiological relationship between excitatory and inhibitory impulses [32]. Decrease in EMG activity was observed after stretching in exercise related cramp victims [33]. Stretching is 
effective in stopping and treating the exercise related cramps $[34,35]$. Non pharmacological means that affect neuromuscular control, e.g. Ice, heat, massage, leg elevation etc may seem relevant by contributing to rest and stretch [36]. The prevention measures would include adaptation training, i.e. preparation of muscle to exercise, by warm ups, well controlled efforts and intermittent rest during exercise.

In the exertional heat cramp, initial localized fasciculation's noticeable only during breaks, indicate imminent major cramps in half an hour or so. Legs are frequently first to manifest these being the most active, implying faster fluid dynamics. Rehydration preferentially restores the plasma volume, reducing thirst and promoting renal loss of free water, even before interstitial fluid compartment is adequately replenished. This would occur particularly if only plain water or low sodium fluid is consumed [23,37,38]. As circulating sodium concentration is maintained, the whole body sodium deficit is not detectable by measuring serum electrolytes, especially upon significant sweat loss by extensive exercise [39]. Better estimate has to be based on sweat sodium loss compared to sodium intake.

At first sign of muscle twitches, prompt oral high salt solution proves effective field strategy (Half a litre of carbohydrate-electrolyte drink containing $3 \mathrm{gm}$ salt taken at once or slowly) [40]. This would allow going on for further hour or so. At subsequent intervals lower-salt fluid may be regularly consumed. If sodium deficit cramps are severe with measurable hypo-natraemia, intravenous rehydration shall be indicated [41].

Athletes suffering exercise related cramp, generally have significant fluid deficits [42]. Restoring body fluids is appropriate preventive measure against occurrence of serious heat illness. Assuming worth of sodium-fluid deficit theory, cramp-prone athletes should add 0.3 to $0.7 \mathrm{~g} / \mathrm{L}$ salt to their drinks [43]. Body weight change monitoring is simplest means to assess need for fluid replacement. It is prudent to resort to prescription of daily salt and fluid intake regimens anticipating the athletic activity undertaken. Individuals with excessive sweating profiles ought to liberally consume high salt solution at regular intervals while engaged in strenuous exercise [44]. The concern is to ensure sodium intake without excess of water for better and faster replenishment of different body fluid compartments [45]. Endurance training expands plasma volume and extracellular fluid compartment [46], and this helps postponing occurrence of neuromuscular fatigue [47].

\section{CONCLUDING REMARK}

Sudden significant localized cramping is often the fatigue type. The sodium deficit cramps grow over minor fasciculation's with wider spread, accompanied by intermittent spasms not relieved by stretching. These would follow severe sweating noticeable from garments. It is noteworthy that athlete may have both kind of cramps requiring keen detection and appropriate address. To prevent exercise related cramps, clinicians should take detailed medical history of each athlete, determine their level of conditioning and monitor their fluid and electrolyte levels. The approach to treating and preventing cramps should be multifaceted as multiple causes are implicated. As most reliable therapy measure, clinician should stretch the cramping muscle. Once the cramp is alleviated, hydration and electrolyte needs must be assessed and addressed. Plyometric exercises that target neuromuscular system may be beneficial to elicit neural adaptations in muscle spindle and golgi tendon organ receptor activity. That may enhance efficiency and sensitivity of reflexive and descending pathways used for neuromuscular control [48]. Because exercise related cramp is a temporarily debilitating condition, prevention is of great interest. It is important for athletes to be well conditioned on return to sports in order to prevent premature muscle fatigue. It is important, however, to use proper progression (intensity, duration, and frequency) during rehabilitation to avoid overstressing the athlete. In combination these factors might decrease the incidence of exercise cramps in athletes during and after functional phases of rehabilitation.

\section{REFERENCES}

1. Braulick KW, Miller KC, Albrecht JM, Tucker JM, Deal JE (2013) Significant and serious dehydration does not affect skeletal muscle cramp threshold frequency. $\mathrm{Br} J$ Sports Med. 47(11): 710-4.

2. Schwellnus MP, Derman EW, Noakes TD (1997) Aetiology of skeletal muscle 'cramps' during exercise: a novel hypothesis. J Sports Sci. 15(3): 277-85.

3. Roeleveld K, Van Engelen BG, Stegeman DF (2000) Possible mechanisms of muscle cramp from temporal and spatial surface EMG characteristics. J Appl Physiol. 88(5): 1698-706.

4. Hutton RS, Nelson DL (1986) Stretch sensitivity of Golgi tendon organs in fatigued gastrocnemius muscle. Med Sci Sports Exerc. 18(1): 69-74.

5. Nelson DL, Hutton RS (1985) Dynamic and static stretch responses in muscle spindle receptors in fatigued muscle. Med Sci Sports Exerc. 17(4): 445-50. 
6. Schwellnus MP (2007) Muscle cramping in the marathon: aetiology and risk factors. Sports Med. 37(4-5): 364-7.

7. Ruff RL (1996) Effects of length changes on $\mathrm{Na}+$ current amplitude and excitability near and far from the end-plate. Muscle Nerve. 19(9): 1084-92.

8. Schwellnus MP (2009) Cause of exercise associated muscle cramps (EAMC)--altered neuromuscular control, dehydration or electrolyte depletion?. Br J Sports Med. 43(6): 4018.

9. Bentley S (1996) Exercise induced muscle cramp: proposed mechanisms and management. Sports Med. 21: 409420.

10. Sulzer NU, Schwellnus MP, Noakes TD (2005) Serum electrolytes in Ironman triathletes with exercise-associated muscle crampingMed Sci Sports Exerc. 37(7): 1081-5.

11. Minetto MA, Holobar A, Botter A, Ravenni R, Farina D (2011) Mechanisms of cramp contractions: peripheral or central generation? J Physiol. 589(Pt 23): 5759-73.

12. Minetto MA, Holobar A, Botter A, Farina D (2013) Origin and development of muscle cramps. Exerc Sport Sci Rev. 41(1): 3-10.

13. Khan SI, Burne JA (2007) Reflex inhibition of normal cramp following electrical stimulation of the muscle tendon. J Neurophysiol. 98(3): 1102-7.

14. Bergeron MF (2003) Heat cramps: fluid and electrolyte challenges during tennis in the heat. J Sci Med Sport. 6: 19-27.

15. McCance RA (1990) Proceedings of the Royal Society of London series B-Biological Sciences, vol.119, 1935-1936: Experimental sodium chloride deficiency in man. Nutr Rev. 48: 145-7.

16. Morgan RM, Patterson MJ, Nimmo MA (2004) Acute effects of dehydration on sweat composition in men during prolonged exercise in the heat. Acta Physiol Scand. 182(1): 37-43.

17. Costill DL, Coté R, Fink W (1976) Muscle water and electrolytes following varied levels of dehydration in man. J Appl Physiol. 40(1): 6-11.

18. Nose H, Mack GW, Shi XR, Nadel ER (1988) Role of osmolality and plasma volume during rehydration in humans. J Appl Physiol. 65(1): 325-31.

19. Bergeron MF, Laird MD, Marinik EL, Brenner JS, Waller JL (1985) Repeated-bout exercise in the heat in young athletes: physiological strain and perceptual responses. J Appl Physiol. 106(2): 476-85.

20. Armstrong LE, Casa DJ, Millard-Stafford M, Moran DS, Pyne SW, Roberts WO (2007) American College of Sports Medicine position stand. Exertional heat illness during training and competition. Med Sci Sports Exerc. 39(3): 556-72.

21. Layzer RB (1994) The origin of muscle fasciculations and cramps. Muscle Nerve. 17(11): 1243-9.

22. Jung AP, Bishop PA, Al-Nawwas A, Dale RB (2005) Influence of Hydration and Electrolyte Supplementation on Incidence and Time to Onset of Exercise-Associated Muscle Cramps. J Athl Train. 40(2): 71-75.

23. Sanders B, Noakes TD, Dennis SC (2001) Sodium replacement and fluid shifts during prolonged exercise in humans. Eur J Appl Physiol. 84(5): 419-25.

24. Nguyen MK, Kurtz I (2006) Whole-body electrolyte-free water clearance: derivation and clinical utility in analyzing the pathogenesis of the dysnatremias. Clin Exp Nephrol. 10(1): 19-24.

25. Fortney SM, Nadel ER, Wenger CB, Bove JR (1981) Effect of blood volume on sweating rate and body fluids in exercising humansJ Appl Physiol Respir Environ Exerc Physiol. 51(6): 1594-600.

26. Eichner ER (2007) The role of sodium in 'heat cramping'. Sports Med. 37(4-5): 368-70.

27. Bergeron MF, Maresh CM, Armstrong LE, Signorile JF, Castellani JW, Kenefick RW, LaGasse KE, Riebe DA (1995) Fluid-electrolyte balance associated with tennis match play in a hot environment. Int J Sport Nutr. 5(3): 180-93.

28. Sjøgaard G, Adams RP, Saltin B (1985) Water and ion shifts in skeletal muscle of humans with intense dynamic knee extension. Am J Physiol. 248(2 Pt 2): R190-6.

29. Felig P, Johnson C, Levitt M, Cunningham J, Keefe F, Boglioli B (1982) Hypernatremia induced by maximal exercise JAMA. 248(10): 1209-11.

30. McGee SR (1990) Muscle cramps. Arch Intern Med. 150(3): 511-8.

31. Jung AP (2006) Exercise associated muscle cramps and functional return to sports Athletic Therapy Today. 2: 4850.

32. Miller KC, Stone MS, Huxel KC, Edwards JE (2010) Exercise-associated muscle cramps: causes, treatment, and prevention. Sports Health. 2(4): 279-83.

33. Helin P (1985) Physiotherapy and electromyography in muscle cramp. Br J Sports Med. 19(4): 230-1.

34. Bertolasi L, De Grandis D, Bongiovanni LG, Zanette GP, Gasperini M (1993) The influence of muscular lengthening on cramps. Ann Neurol. 33(2): 176-80.

35. Wagner T, Behnia N, Ancheta WK, Shen R, Farrokhi S, Powers CM (2010) Strengthening and neuromuscular reeducation of the gluteus maximus in a triathlete with exercise-associated cramping of the hamstrings. J Orthop Sports Phys Ther. 40(2): 112-9.

36. Blyton F, Chuter V, Walter KE, Burns J (2012) Non-drug therapies for lower limb muscle cramps. Cochrane Database Syst Rev.1: CD008496.

37. Mitchell JB, Phillips MD, Mercer SP, Baylies HL, Pizza 
FX (2000) Post exercise rehydration: effect of $\mathrm{Na}(+)$ and volume on restoration of fluid spaces and cardiovascular function. J Appl Physiol .89(4): 1302-9.

38. Nose H, Mack GW, Shi XR, Nadel ER (1988) Shift in body fluid compartments after dehydration in humans. J Appl Physiol. 65(1): 318-24.

39. Vaamonde CA (1982) Sodium Depletion, in Paper S Ed, Sodium: Its biological significance. Boca Raton. CRC Press. 207-234.

40. Bergeron MF (1996) Heat cramps during tennis: a case report. Int J Sport Nutr. 6: 62-68.

41. Siegel AJ (2007) Hypertonic (3\%) sodium chloride for emergent treatment of exercise-associated hypotonic encephalopathy. Sports Med. 37(4-5): 459-62.

42. Stofan JR, Zachwieja JJ, Horswill CA, Murray R, Anderson SA, Eichner ER (2005) Sweat and sodium losses in NCAA football players: a precursor to heat cramps?. Int J Sport Nutr Exerc Metab. 15(6): 641-52.

43. Binkley HM, Beckett J, Casa DJ, Kleiner DM, Plummer
PE (2002) National Athletic Trainers' Association Position Statement: Exertional Heat Illnesses. J Athl Train. 37(3): 329-343.

44. Bergeron MF (2007) Exertional heat cramps: recovery and return to play. J Sports Rehab. 16: 190-196.

45. Maughan RJ, Leiper JB (1995) Sodium intake and post-exercise rehydration in man. Eur J Appl Physiol Occup Physiol. 71(4): 311-9.

46. Convertino VA (1991) Blood volume: its adaptation to endurance training Med Sci Sports Exerc. 23: 1338-48.

47. Häkkinen K, Komi PV (1985) Fatiguability in voluntary and reflex contraction after conditioning of human skeletal muscleElectromyogr Clin Neurophysiol. 25(5): 319-30.

48. Wilk KE, Voight ML, Keirns MA, Gambetta V, Andrews JR, Dillman CJ (1993) Stretch-shortening drills for the upper extremities: theory and clinical application. J Orthop Sports Phys Ther. 17(5): 225-39. 